\title{
Implications of Synchronous IVR Radio on Syrian Refugee Health and Community Dynamics*
}

\author{
R. Talhouk \\ Open Lab \\ United Kingdom \\ R.R.Talhouk2@ncl.ac.uk
}

\author{
S. Mesmar \\ American University of Beirut \\ Lebanon \\ Smm46@mail.aub.edu
}

\author{
M. Najem \\ American University of Beirut \\ Lebanon \\ Mn17@aub.edu.lb
}

\author{
T. Bartindale \\ Open Lab \\ United Kingdom \\ Tom.Bartindale@ncl.ac.uk
}

\author{
C. Akik \\ American University of Beirut \\ Lebanon \\ Ca36@aub.edu.lb
}

\author{
H. Ghattas \\ American University of Beirut \\ Lebanon \\ Hg15@aub.edu.lb \\ M. Balaam \\ Open Lab \\ United Kingdom \\ Madeline.Balaam@ncl.ac.uk
}

\author{
K. Montague \\ Open Lab \\ United Kingdom \\ Kyle.Montague@ncl.ac.uk
}

\author{
A. Ghassani \\ Amel Association \\ Lebanon \\ Aghasani@gmail.com \\ P. Olivier \\ Open Lab \\ United Kingdom \\ Patrick.Olivier@ncl.ac.uk
}

\begin{abstract}
With 1,033,513 Syrian refugees adding a strain on the Lebanese healthcare system, innovation is key to improving access to healthcare. Our previous work identified the potential for technology to improve access to antenatal care services and increase refugee agency. Using (1) paper mock ups and a mobile based prototype, (2) process mapping, (3) focus groups and interviews and (4) key informant meetings, we explored the concept of refugee led community radio shows to deliver peerled healthcare. We observed the influence of community radio
\end{abstract}

\section{(c) (1) (2)}

shows on Syrian refugee health education, community dynamics and community agency in relationships between healthcare providers and refugees. Refugees were positively impacted through situating the technology within the community. We highlight issues around trust, agency, understanding, selforganization and privacy that resulted from running the shows through mock ups and a mobile based prototype. Our findings inform future work in community run radio shows.

\section{CCS CONCEPTS}

- Human Centered Computing $\rightarrow$ Human Computer Interaction $\rightarrow$ Empirical Studies in HCI

\section{KEYWORDS}

Refugees; Syrian Refugees; Lebanon; IVR; Technology; Community Radio Shows; Agency; Health Education;

\section{ACM Reference format:}

C\&T '17, June 26-30, 2017, Troyes, France (c) 2017 Copyright is held by the owner/author(s) ACM ISBN 978-1-4503-4854-6/17/06. http://dx.doi.org/10.1145/3083671.3083690
R. Talhouk, T. Bartindale, K. Montague, S. Mesmar, C. Akik, A. Ghassani, M. Najem, H. Ghattas, P. Olivier and M. Balaam. 2017.Implications of Synchronous IVR Radio on Syrian Refugee Health and Community Dynamics. In Proceedings of the $8^{\text {th }}$ International Conference on Communities and Technologies, Troyes, France, fune 2017 (C\&T'17), 10 pages. DOI: 10.1145/3083671.3083690 


\section{INTRODUCTION}

The protracted nature of the Syrian refugee crisis calls for innovative solutions. This is especially true in Lebanon, a country that hosts over 1 million Syrian refugees [25]. Lebanon's Crisis Response Plan prioritizes improving refugee access to healthcare services, including outreach and health education. Previous exploration of access to antenatal care by Syrian refugees residing in Informal Tented Settlements (ITSs) in rural Lebanon revealed several barriers to delivering care. First that a lack of transportation to primary healthcare clinics (PHCs) discourages refugees from seeking care, and second that refugees characterized their relationship with healthcare providers (HCPs) to include miscommunication and negative attitudes. In conclusion, they felt they had low agency in communicating their health concerns [23]. Refugee women indicated a need for new modes of direct communication with HCPs, however viewed HCP reluctance to engage with them using technology to be a major barrier [23]. The study indicated that interactions through digital mediators should be configured to increase refugee agency by allowing them an active role in communicating with HCPs. This is made possible by the fact that almost all refugee households have at least one mobile phone and access to the internet through Wi-Fi and/or 3G. However, Wi-Fi networks were considered too slow to support video communication and $3 \mathrm{G}$ tariffs are prohibitively high [23].

Work conducted in spaces for refugee creativity [28,30] and the use of co-design methods with refugees [9] have highlighted the potential for refugees to have a more proactive role in the use of technology. However, recent technological deployments have focused on one-way dissemination of information to refugees $[2,4]$. Similarities in the technology constrained settings of Syrian refugees in Lebanon and that of marginalized communities in developing countries allow for the adaptation of Human Computer Interaction (HCI) for development technologies to address Syrian refugee needs. An example of such a technology is synchronous Interactive Voice Response (IVR), previously deployed in India, allowing for health workers to run radio shows that can be accessed through mobile devices over the data network [13]. Given refugees expressed an interest in using technology more proactively in engaging with HCPs [23] and the possibility of synchronous communication to open up dialogues for shifting agency within the refugee-HCP relationships, we wanted to extend this work [13]. We did so by exploring how community radio shows hosted by refugees may impact refugee agency. Consequently, we designed Citizen Radio (CR), a synchronous platform for delivering community talkshow style radio hosted by refugee community members rather than health workers. We provide insight into the implications of running these shows on HCP-refugee relationships and community dynamics.

Initially our study was designed to train a refugee to use $\mathrm{CR}$ and deploy radio shows within her community. However, upon prototype testing of $\mathrm{CR}$ in rural Lebanon, we found that more work was required to maintain a consistent listener experience when operating on the internal Lebanese telephone network.
Restrictions in the network placed on concurrent calls resulted in $\mathrm{CR}$ only reliably maintaining a connection with a maximum of three mobile phone lines simultaneously. This restricted us from fully deploying $\mathrm{CR}$ with multiple listeners participating from the privacy of their tents. However, given the commitments we had made to put the community in contact with HCPs to whom they can ask their health-related questions, we adapted the study design such that listeners congregated to listen from one tent, using a single phone and paper mock ups (to simulate features like queueing and tagging of content to generate metadata) while the HCP guest participated remotely. This allowed us to explore the concept of health community radio shows situated within refugee communities, and observe the contextual factors related to the introduction of the technology, such as changes in community power dynamics.

\section{RELATED WORK}

\subsection{Human Computer Interaction \& Refugees}

Deployments in HCI research conducted around refugees explored the use of technology to facilitate resettlement. Brown et al. [4] utilized asynchronous IVR, placing a translator who shared the same culture and language of the refugee as a mediator on a call with the refugee. Similarly, Baranoff et al. [2] placed near field communication tags around a city to transmit informational messages in the appropriate language to refugees and to notify their social workers when health appointments were attended. Although these designs [2,4] place technology in the hands of refugees, there remains space in their designs for more grass-root approaches to using technology in improving access to services. Other initiatives have included refugee populations in more proactive roles. Participatory community mapping of refugee camps has been theorized to contribute to strengthening the community [29]. Likewise, computer clubs in Palestinian refugee camps engaged youth in capacity building activities and digital role playing $[28,30]$.

\subsection{Telephony in Resource Constrained Settings}

IVR technologies have become increasingly popular in low resource settings due to their low cost and potential for scalability [7]. IVR has been successfully used to engage communities in shared discussions around community issues, agriculture, and employment $[6,18,20]$. In India IVR has been used as a tool for citizen journalism; CGNet Swara allows community members to record/listen to journalist moderated audio clips from a content store through phones and a website [18]. RootIO, deployed in Uganda, utilizes IVR to allow for free calls to be made with questions that are later aired live on radio shows that are run and managed by small agricultural communities. Nevertheless, the bulk of the content is generated centrally by a partner organization [6]. Similarly, Avaaj Otalo [19] was used to broadcast agricultural community radio shows where the show producer would incorporate concerns shared through voice calls into the development of the show. Taking 
Implications of Synchronous IVR Radio on Syrian Refugee Health and Community Dynamics

this further, Mobile Vaani allows listeners to comment on audio clips and share content with others [16] to sustain a dialogue within the community. However, there are few examples of IVR supporting health directly. iCope [8] allows patients to access health guidelines through telephony and Project Health Line, piloted in Pakistan, supported health workers through asynchronous responses to health questions [22]. Although community IVR-based shows increase community agency in contributing content, the presence of moderators, needed to make the pre-recorded content easy to navigate by listeners, can be a restrictive process [27]. Sangeet Swara addressed this by supporting community moderation, where user-generated ratings of audio clips dictate the order in which clips are played to other listeners [26]. However, Kazakos et al.'s [13] deployment of a synchronous IVR system eliminated the need for post-production content moderators as listeners are participating live in the shows. Their platform [13] allows for social workers to have HCPs as guest speakers on a radio-like telephony broadcast with listeners joining the call to ask questions. The system explored the potential for synchronous IVR to become a more interactive platform for creating spontaneous and responsive health dialogues. However, content was specified and delivered by a health worker in a top-down dissemination approach indicative of these broadcast platforms. Little is known about how communities would organize grassroot community shows and the changes in community dynamics and relationships with HCPs that may ensue upon situating the technology within the community.

\section{CITIZEN RADIO}

We developed $\mathrm{CR}$ as an initial prototype to explore the considerations that arise when situating synchronous IVR technology within the community. Free of charge for participants, CR connects a host, a guest speaker and multiple listeners through their mobile phones as if they were listening to a traditional radio talk show. A mobile application appropriate for semi-literate users was provided for the host and audio prompts were used to provide the host and listeners with information on the format of the show. While the show was running, the interface provided the host a simple visualization of the show structure, time elapsed, the upcoming segment of the show, number of listeners and callers and the connection status of the guest speaker participating remotely. The host could allow callers to ask their questions on air and play audio she had preprepared using the interface (e.g. to advertise the next show). The platform was designed to scaffold the delivery of a talkshow style broadcast with two subtopics of conversation and a Q\&A segment for each subtopic. If a listener indicates they want to ask a question during a show, the system would ask them to select from an audio menu the subtopic their question pertains to, thus tagging their question and generating metadata that can be used to identify the question and response audio for later use. Communication between the host's mobile application and the
C\&T'17, June 2017, Troyes, France

CR cloud based platform (FreeSwitch and Python application) was performed over an intermittent data connection, and is intended to be resilient against both Internet and telephony dropout. Listeners were dialed into the radio show using the existing PTSN (telephone) network. Despite adapting the system to cater for telephone network connectivity issues experienced (using auto-dial-back and multiple providers), inherent inconsistencies in the Lebanese telephony network restricted us to having only one listener device, a host and a remote guest. We are currently working with the relevant ministries in Lebanon to address the restrictions placed on the system for further deployments. However, in response to the telephony restriction and obligations to deliver much needed health information to the community, we adapted our study. Paper mock ups were used to mimic the functions of the show, such as tagging of questions and caller queuing.

\section{METHODS}

The study was conducted over two months, primarily in an ITS in rural Lebanon, during which the researchers engaged extensively with stakeholders and the refugee community. Ethical approval was obtained from two ethics committees. The study was conducted by two native Arabic speakers that were part of the research team.

\subsection{Recruitment}

Our previous work [23] with refugee women in ITSs in rural Lebanon had identified a community (84 individuals residing in 20 tents) that (1) is a long walk from the nearest PHC, (2) had voiced an interest in a digital medium to communicate with HCPs and (3) had extensively reflected on their low agency within their relationships with HCPs and its effect on their antenatal health. After an initial introductory engagement with the women we obtained informed consent from those interested in participating ( $\mathrm{n}=15$; age range: $18-60$ years). We then identified and obtained informed consent from the host by asking the women whom among them is usually sought out for health advice. Three female HCPs (two gynecologists and one midwife) were recruited to be guests on the show through a local non-governmental organization (NGO) that runs PHCs in the region. Additionally, a meeting was conducted with key informants from the Ministry of Public Health (MoPH) for feedback.

\subsection{Selection of Show Health Topics}

Community engagements identified that the women had questions regarding healthy diets and vaginal infections. Furthermore, the HCPs identified the common health conditions encountered in their clinics. Accordingly, the following topics were selected to meet the interests of both the refugees and HCPs: (1) family planning, (2) nutritional supplements and emergency cases, (3) healthy pregnancies and (4) a healthy diet and personal hygiene. Per the MoPH's request, the health 
information disseminated during the shows, was reviewed for accuracy by a medical consultant specialized in reproductive health.

\subsection{Data Capture \& Analysis}

Throughout we collected observational notes on the community dynamics that resulted from the introduction and running of the shows. The host was introduced to the CR mobile application to familiarize herself with it. This also contributed to the in-country testing of the platform that revealed local telephony restrictions. Only one host was selected so that we could observe changes in her interactions with HCPs and community members over time. Four activities were then conducted to meet the study's aims:

4.3.1 Activity 1: Exploring Agency and Community Dynamics in Relation to Commissioning of Community Radio Shows. The host and a community member were asked to map out, using cardboard sheets and colored markers, how they would go about commissioning a community radio show (initiating and deciding content). This activity unpacked the nuances of the refugee-HCP relationship and community dynamics by posing hypothetical questions on how participants would approach HCPs and community members to start a community radio show (discussions were audio recorded).

4.3.2 Activity 2: Exploring the Running of Community Radio Shows. Community radio shows were conducted by combining paper mock ups and the limited working functionality of the CR prototype. A listener phone was placed in the middle of a tent and women, who were seated in a circle, would take turns in asking their questions (figure 1). The first show was conducted as the host had envisioned it. She designed and made a queuing system based on traffic light colored cards to be used in the Q\&A segments. Three traffic light colored paper cards (red, yellow and green) were used with emoji drawn on them to represent the queue to ask questions. Based on the request of the host, the first two shows were conducted with the host seated among the women. Consequently, the application was not used during these shows, and the host while seated among the women made a simple phone call to the guest HCP. The third and fourth shows were mediated by $\mathrm{CR}$, where the host was in a separate tent, the guest HCP was dialed in remotely and as in previous shows the women would sit in a circle around the listener phone. In addition, the research team requested that shows two, three and four, should mimic the real time tagging of the questions by the listeners. To do so listeners were given different colored cards each color indicating a subtopic - and were asked to raise that card to request a turn in the questions queue.

4.3.3 Activity 3: Evaluating Experiences of Participating in Shows. Evaluations of experiences of participating in the shows were collected through five audio recorded focus groups and three interviews with the women and HCPs, respectively. Focus groups with the women were conducted after each show, probing at interactions the research team had observed during the show. One focus group was conducted at the end of the study where women provided general feedback regarding community radio shows.
4.3.4 Activity 4: Evaluating the Potential Use of Citizen Radio. A meeting was conducted with key informants within the $\mathrm{MoPH}$ and our collaborating NGO. The discussions explored the potential uses of CR within the health sector in Lebanon.

All qualitative data were directly translated and transcribed in to English by the lead author, who is a native Arabic speaker. Thematic analysis [3] was conducted. Emergent themes were classified according to the study's research questions and they were validated by re-discussing them with participants.

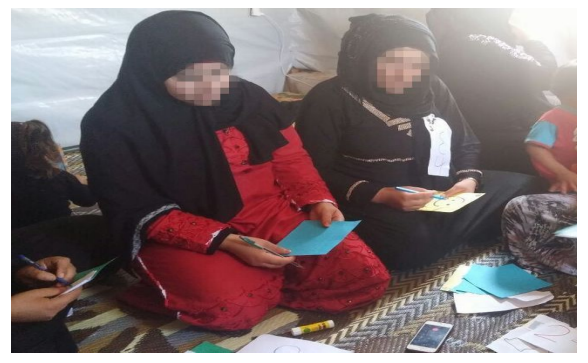

Figure 1: Women participating in Activity 2, engaging with the HCP through the listener phone and taking notes

\section{FINDINGS}

The study revealed changes in community dynamics and in the HCP-refugee relationship (agency, trust and understanding), and complexities related to privacy and health education and outreach.

\subsection{Running a Community Radio Show}

During activity 2, insights around self-organization and privacy were revealed in addition to data surrounding changes in the HCP-refugee relationship and host/listener interactions (with and without support from $\mathrm{CR}$ ).

5.1.1 Self-Organization. Women showed a high degree of selforganization during the running of the shows. Initially the host distributed the queuing cards but eventually women were moving the cards around on their own. They would also whisper their questions to each other to avoid duplication of questions. Lastly, as the host was initially preoccupied with keeping track of the queueing cards the other women took on the role of managing noise and attention of others. When side conversations would get too loud the other women would silently indicate to them to stop talking. The women's selforganization was consistent even in the shows that were mediated by CR, with the host not co-located.

5.1.2 Privacy. Initially, women expressed that they had no issues with participating in the show as a group using one listener phone. One woman said, "We are all women and if one of us has a health problem she won't be ashamed" [ $\left.W_{N}\right]$. However, this perspective changed after an incident during show two. Before the beginning of show two, an older woman $\left(\mathrm{W}_{\mathrm{M}}\right)$ entered the tent and gave consent to participate in the study. During the show, we observed that some of the women that were usually more engaging with the HCPs were silent. 
Implications of Synchronous IVR Radio on Syrian Refugee Health and Community Dynamics

Furthermore, $\mathrm{W}_{\mathrm{M}}$ was sitting in a corner causing disruptions. Unlike the usual practice of other women quieting down the disturbance, they allowed her to disrupt the show and tell the $\mathrm{HCP}$, "This is taking too long let us end the show now" $\left[W_{M}\right]$. At a later point when the women continued to ask the HCP questions $\mathrm{W}_{\mathrm{M}}$ threatened "I will tell your husbands what you are doing" and the women refrained from asking questions, thus resulting in less questions being asked in show two (table 1). After the show ended, the women informed us that $\mathrm{W}_{\mathrm{M}}$ holds a strong matriarchal position within the community and the women she was chastising for asking questions were her great daughters inlaw. They elaborated that the women were not as engaged with the HCP because they did not want to disclose anything in front of $\mathrm{W}_{\mathrm{M}}$ that might cause familial disputes. They indicated that restricting invitations to participate in the show based on age would be a way to respectfully exclude her from the study. However, they made sure that the exclusion should be done in a way that would not exclude other older women they trust and consider key members of the community by informing them that they can still participate but not advertise their participation. Other participants also started stating that the original model of CR would be ideal in these cases as each woman can participate on her own from her tent.

5.1.3 Community Agency within HCP-Refugee Relationship. Women displayed a high level of engagement with the HCPs. During the Q\&A segments they would all move closer to the phone making the circle smaller. They would also take notes of what the HCP was saying - more so when the HCP was answering their questions. Women would recount their health ailment related to the topic and build up to a question specific to their case. In one example, $\mathrm{W}_{\mathrm{Ro}}$ went into detail regarding her menstrual cycle when asking about the appropriate contraceptive method, "I have tried to take the contraceptive pills but I'm starting to get an irregular period and it's been three months that I haven't got my period but there is no pregnancy". There were also instances in which an answer by the HCP would be followed with a follow-up question by the same woman. During the first show the women sometimes asked questions unrelated to the topic of the show and the HCP responded by saying "even though this question is off-topic I will answer it" $\left[H C P_{1}\right]$. Afterwards, they would ask permission from the HCP to ask an off-topic question. A total of 33 questions were asked across the four shows (Table 1). Women asked less questions in show four possibly because the HCP provided lengthy answers as compared to the other HCPs.

5.1.4 Host \& Listeners Power Dynamics. The host played an influential role while running the show. In the shows where she was co-located with the listeners she would point at women encouraging them to ask questions. This is especially true for the women that she knew were suffering from health issues related to the topic at hand. During the show, she would write down questions she thought should be asked and would pass them on to the other women to ask. When asked why she wouldn't ask those questions herself she said "because I am the host, I don't
C\&T'17, June 2017, Troyes, France

want to confuse people" $\left[W_{H}\right]$. She also refused to be given a participant number and reiterated that she is the host and would not need a participant number like the listeners. However, when she was not seated with the other women during the last two shows, and was unable to pass questions on to them, she chose to ask questions herself.

Table 1: The number of questions varied per the interest of the women in the topic.

\begin{tabular}{ccc}
\hline & Number of Listeners & $\begin{array}{c}\text { Number of } \\
\text { Questions }\end{array}$ \\
\hline Show 1 & 10 & 12 \\
Show 2 & 9 & 4 \\
Show 3 & 8 & 14 \\
Show 4 & 11 & 5 \\
\hline
\end{tabular}

During the first show the host also played a role in organizing the question queue. Furthermore, she would make sure no one would talk out of turn, she would physically move closer to the phone blocking access to others when she felt a woman was going to speak out of turn. In several instances, she would see a woman begin to talk without being in the queue and she would immediately say "Ok we have another question" $\left[W_{H}\right]$ and point to the woman whose turn it was to ask a question. The host struggled during the shows mediated by $\mathrm{CR}$ without the visual cues from the women during the Q\&A segments. Even though the women were self-organizing themselves when they wanted to ask questions, she felt the need to try to peek into the other tent to see who was going to ask the next question. She maintained control throughout by saying "We have another question, please go ahead" $\left[W_{H}\right]$. Furthermore, the host's higher agency over that of the listeners was observed when they refrained from asking the HCP for clarification and instead asked the host to ask the HCP to repeat what they had not understood.

5.1.5 Host \& Guest Power Dynamics. While the host had the confidence to organize the women's questions the HCP's position of power was still dominated when the host initially communicated with them. She would wait for the HCP to finish disseminating the health education segment and then allow the women to ask their questions regardless of the time elapsed. When she felt that the guest has gone over-time, she would look at the research team for re-assurance and ask if she should cut the HCP off and transition to the Q\&A segments. However, as the shows progressed and with $\mathrm{CR}$ showing the time elapsed, she gained the confidence to transition to the Q\&A segments without seeking re-assurance. Furthermore, since CR calls the guest first and then the listener phone, there was some time in which only the host and the guest were on the show. In the third show (the first supported by CR), the host had some difficulty conversing with the HCP while the system brought the listener phone into the call, however she did inform the HCP that she was talking too quickly for them to understand. During the 
fourth show, the host was more confident and aware of the initial time lapse; therefore, she used that time to thank the HCP for participating and to inform her of the number of women participating. She also began giving the HCP instructions by saying "I want to organize with you before we start. We will start with the first topic and not continue to the second. We take a break for questions and then go to the second topic".

\subsection{Shifts in Relationships}

The participants considered the health community radio shows to be successful. Changes in agency, trust and understanding within relationships and communication were evident.

5.2.1 Communication. The listeners expressed that communicating with HCPs through the shows had benefits over face-to-face consultations. One participant explained that "Doctors at the clinic don't let us ask anything $\left[W_{N}\right]$ ". Additionally, the remote participation of the HCPs made the listeners feel more comfortable in asking their questions and voicing their concerns, as $\mathrm{W}_{\mathrm{J}}$ stated that this mode of communication is better because "when I go to the doctor I forget everything". The remote nature of communication was also found to be beneficial by the HCPs. They emphasized that CR allowed them to connect with the communities remotely without having to travel to remote ITSs and therefore giving them the ability to connect with more communities. Additionally, one $\mathrm{HCP}$, that is not experienced in delivering health education to large audiences, explained that the remote yet real-time nature of her participation made her less nervous. She emphasized that "I wasn't nervous because I didn't see them and they did not see $m e$ " $\left[\mathrm{HCP}_{3}\right]$. She hoped that by participating in these shows she would gain the experience to better communicate with refugees.

When compared to health education given at clinics, participants stated that listening to each other's questions was highly beneficial. $\mathrm{W}_{\mathrm{N}}$ also explained that the community radio show experience differed from clinic consultations and health lectures because during the show she felt that the "doctor understood us and took time to explain to us". The HCPs also found the interaction to be highly responsive. $\mathrm{HCP}_{2}$ explained that "I really didn't expect them to interact, concentrate and understand to this degree". Furthermore, HCPs compared the shows to other one-to-many education modalities (health education lectures and TV shows). They indicated that CR was more engaging than the afore mentioned mediums due to its synchronous and interactive nature. They highlighted that in comparison refugees are not usually as responsive during faceto-face health education lectures. Additionally, the live nature of CR put them in closer contact with the community, something HCPs had not experienced when participating in TV talk shows.

5.2.2 Trust and Understanding. Both the community and HCPs reflected on an increase in trust and understanding within their relationship. The trust the women built with the HCPs was prevalent, so much so that they requested the names of the HCPs (which had been kept anonymous) to possibly visit them in their clinics. $\mathrm{W}_{\mathrm{J}}$ indicated that "I feel that this HCP understands us more than others". The trust built between the listeners and the HCPs allowed the women to consider forgoing visiting the clinic to ask their health questions. They all agreed that the HCPs' advice was reliable. This was true for advice regarding nutritional supplements and non-medicinal management of antenatal ailments such as heartburn and nausea. For cases where the HCPs recommended they visit a clinic for medicinal treatments, the women indicated that they would follow these instructions. $\mathrm{W}_{\mathrm{Ha}}$ explained regarding another participant related to her, "Now we know that when she is pregnant we should take her to the doctor to change her asthma medication". Trust was also built between the HCPs and the host as they emphasized the vital role of the host within this engagement. HCPs indicated that they relied on her to organize the show, "she was the main person communicating with me and she organized the questions and was very aware about the topic" $\left[\mathrm{HCP}_{2}\right]$. This effect extended to the community in the form of HCPs trusting that the community wants to actively attain better health. Participating in the community radio shows gave the HCPs a better understanding of the community, "I now know that their situation is what holds them back. I put myself in their shoes...I realized that there are a lot of them that are health aware" $\left[\mathrm{HCP}_{3}\right]$.

5.2.3 Opinions Regarding Potential of $C R$. The potential of community radio shows was recognized by the various individuals and groups involved. The women saw value in editing and reusing the audio recordings from the shows. "There was something the doctor was saying but I got distracted if I had recordings $I$ could listen to it again later" $\left[W_{H}\right]$. They also indicated that recordings could be of use to other communities and other women within their community that missed a show. We observed the host answering the question of a woman who could not attend the show based on information disseminated during the show. HCPs identified the potential for show recordings to contribute to the training of other $\mathrm{HCPs}$. $\mathrm{HCP}_{1}$ highlighted that these recordings could be used as examples for recent graduates on how to provide education for refugee and low literacy patients; "by listening to the shows they can learn how to speak to patients in a way they understand" $\left[\mathrm{HCP}_{1}\right]$. The $\mathrm{MoPH}$ expressed that the potential of community radio shows could be amplified by editing and reusing recordings from the shows and sending them as audio messages to communities. Furthermore, to ensure that the health education being disseminated is up to standard, they suggested the use of CR to train health educators and disseminate clinical guidelines. It is important to note that they emphasized the need for $C R$ to support the validation of the health material being disseminated through the show.

\subsection{Commissioning a Community Radio Show}

By exploring how a host would go about initiating a community radio show we unpicked the community dynamics that would affect community participation in the shows and vice versa. We also shed light on considerations for host-HCP relationships.

5.3.1 Host Agency. Our observations showed that the role of host was considered prestigious and brought with it an increase in agency within the community. Both the currently selected host $\left(\mathrm{W}_{\mathrm{H}}\right)$ and the participant $\left(\mathrm{W}_{\mathrm{O}}\right)$ engaging in Activity 1 identified that, if they were to be the ones initiating the process 
Implications of Synchronous IVR Radio on Syrian Refugee Health and Community Dynamics

of commissioning a show then they should be the host. The current host explicitly pointed out "I will host the show because it was me who started talking with the women" $\left[W_{H}\right]$. The other women in the community acknowledged the host's newly found power. This was observed when Wo was cautious in her questioning of who would govern communicating with the other women when commissioning a show. She cautiously whispered in front of the current host "but who would oversee this WhatsApp group [to be used in the topic selection process]?" [ $\left.W_{O}\right]$. The host's increase in agency was also associated, per the women, with the host's relatively increased contact with the research team. During the initial visits to the ITS the research team would make sure to engage with all the women however substantial time was spent alone with the host in her tent to familiarize her with CR. This created some tension that the host explained to us "first the women were not very enthusiastic ... you spent a lot of time alone with me. But when we started doing group activities they started looking forward to you coming" $\left[W_{H}\right]$.

5.3.2 Community Implications of Host Selection. Our observations revealed that the selection of the host influences community dynamics. Our selection of the host, led to the mixing of sub-communities within the ITS that was not always present. The community consists mainly of families originating from Village $S$ in rural Syria. The rest of the families in the settlement were scattered from across Syria. A woman from Village S explained to us that "We do not go to the other side of the settlement, because they [families on the other side] are not from among us" $\left[W_{R}\right]$. Although the host is not from Village $S$ the other women were comfortable going to her tent to conduct the shows because her husband is always at work and she is very welcoming to all the women in the ITS. The host not being from Village S also allowed women from the other side of the ITS, that are also not from Village $S$, to feel comfortable participating in the shows. Consequently, her tent became a meeting point where women from both sides of the ITS felt comfortable discussing their health. Although, the selection of the host brought together different sub-communities, her selection alienated her in-laws. Due to a familial dispute she has with them, they withdrew their participation in the study upon her selection as host.

5.3.3 Community Agency in Topic \& Show Time Selection. Both $\mathrm{W}_{\mathrm{H}}$ and $\mathrm{W}_{\mathrm{O}}$ in Activity 1, considered the selection of show topic, date and time to be essential to the community. They indicated that topics would be selected through discussions [ $\left.\mathrm{W}_{\mathrm{O}}\right]$ or voting on topics $\left[\mathrm{W}_{\mathrm{H}}\right]$ with the other women. Community enthusiasm in selecting topics was observed as they constantly suggested to us various topics for future shows. Including HCPs within the topic selection process was not indicated by $\mathrm{W}_{\mathrm{H}}$ and $\mathrm{W}_{\mathrm{O}}$. Given that a HCP is already selected, $\mathrm{W}_{\mathrm{H}}$ said she would visit the $\mathrm{HCP}$ to communicate the selected topic whereas $\mathrm{W}_{\mathrm{O}}$ explained that she would communicate with the HCP via WhatsApp.

The selection of the time to air the show proved to be more controversial than expected. The women's husbands played a
C\&T'17, June 2017, Troyes, France

role in influencing the time at which the women preferred the show to air. We observed some women join the shows and leave in a rush. Upon further probing, $\mathrm{W}_{\mathrm{N}}$ explained that her husband was getting upset when she left their children in his care and therefore was pressuring her to withdraw her participation. Therefore, during the activities around commissioning, both $\mathrm{W}_{\mathrm{H}}$ and $\mathrm{W}_{\mathrm{O}}$ expressed the need to find a time for the shows when the husbands are more likely to be at work or asleep. Like the topic selection processes, they did not consider the HCPs' schedules for the show time.

\section{DISCUSSION}

Our findings allow us to examine and extrapolate the potential of community radio shows hosted by refugees in providing health education, increasing understanding and trust between HCPs and refugee communities, and increasing the agency of refugee communities. While findings regarding self-organization and privacy may not apply in the case of a fully functioning CR, the findings related to changes in $\mathrm{HCP} /$ refugee relationships and community dynamics may inform future designs of $\mathrm{CR}$ and its situation in refugee settlements.

\subsection{Community Radio Shows for Health}

The consistent engagement of the refugee participants in the shows and the positive feedback from the community, HCPs and stakeholders involved indicates the success of community radio shows in health outreach and education. Similar to tools developed to support health/social workers in their work [14,17], the HCPs and MoPH indicated that the community radio shows and the recordings generated from them can be used to train healthcare providers in health education. Furthermore, a key distinction from Sehat Ki Vaani [13] was that the host was able to present the show in a different location than the guest. The remote participation of the HCPs allowed them to provide health education free of the anxiety associated with presenting to large audiences. Additionally, as would be expected, decreased the effort and time needed to go to the ITSs. Finally, the one-tomany model provided through CR gave a wider access to hardto-reach communities, and with higher responsiveness than traditional health dissemination modalities currently in use.

Lastly, a report on technologies designed for refugees calls for stringent governance of content being disseminated through technology as wrong information could be harmful [10]. This is especially true for health information. Any design that allows for the participation of multiple HCPs should find a balance between the spontaneous nature of shows and the validation of information disseminated without interfering with the synchronous nature of the shows. There is room to do so by making the shows and the recordings resulting from them visible to the public and regulatory bodies that ensure quality of healthcare services. 


\subsection{Community Radio Shows \& Community Dynamics}

The selection of the host had several implications regarding participation and community dynamics. The introduction of the community radio shows with $\mathrm{W}_{\mathrm{H}}$ as the host brought together previously divided factions of the community. However, it also restricted participation of individuals who avoided interacting with the host. Additionally, the shows spurred discussions in the community regarding who would oversee the shows. In contrast to the deployment of Sehat Ki Vaani [13], that indicated the need to support the host in managing callers, the refugee listeners portrayed a high level of self-organization that can be leveraged to decrease the burden on the host. Our data suggest that taking on the role of the host increased $\mathrm{W}_{\mathrm{H}}$ 's agency within her community. Similarly, the use of participatory mapping by communities has also identified that it is the community facilitators that become more empowered by their higher degree of control over the technology [5]. The host's agency allowed her to encourage the other women to participate. However, in instances when women began to show their own agency (e.g. self-organizing the Q\&A segments) the host would re-iterate her control over the show through multiple interactions with the listeners (passing on of questions, cutting off listeners and refusal to have a participant number). The conflict between the host's agency and the listeners' self-organization calls for consideration of the impact of community radio shows on the power dynamics within a community. The potential for tensions to arise, consequent to the host being the focal point of the technology, was echoed in the women's dislike of the research team spending more time with the host. We recommend careful consideration of how technology is distributed among community members (i.e. who is in control of the technology and the ownership of the radio show initiation and management). Future designs should allow for community members to negotiate roles amongst themselves.

\subsection{Community Radio Shows for Health Agency}

Technologies designed to be used by refugees [2,4] still maintain a top-down approach to disseminating information. Literature [11] and our findings indicate that the HCP-patient relationships are characterized by low patient agency. However as theorized by Talhouk et al [23], participating in shows mediated by refugees increased community members' health agency. Additionally, the synchronous nature of the communication allowed for spontaneous conversations that improved their communication with HCPs and their confidence in doing so.

While RootIO community radio shows are hosted by NGOs with community volunteers acting as presenters [6], our findings showed that refugee communities are capable and willing to both (1) contribute topics they would like to learn about, and (2) to host health community radio shows. However, our findings showed that such designs should support refugee members in addressing the practical challenges they may face. For example, providing appropriate support for the community to identify a guest $\mathrm{HCP}$ and negotiating a time for the show. We recommend future designs support network creation between refugee communities, HCPs and organizations that are willing to participate. The building of such a network could be mediated through technologies refugees are already familiar with, such as WhatsApp. We also found the increased confidence of the host decreased the need for audio fillers, which were incorporated to support her during "black holes" [13]. Her new-found agency allowed her to even give the HCP instructions and information on how she would be hosting the last show.

The remote communication through $\mathrm{CR}$ provided a space for both HCPs and community members devoid of the usual communication barriers experienced in face-to-face communication. Previous findings within the context of refugees and antenatal health [23] have indicated a power dynamic in favor of HCPs that is in line with international literature on HCP-patient interactions [11]. Our study showed that through community radio shows refugees could ask questions that they were not comfortable asking in clinical settings. The large volume of questions asked and the presence of spontaneous follow-up questions are further evidence of the refugees overcoming their reported low agency. This shift in agency has not been reported in previous technologies designed to be used and managed by health and social workers in developing countries [14,24]. Our findings provide a basis for more rigorous studies to be conducted that compare HCP-refugee communication in face-to-face settings versus remote synchronous communication. Lastly, we believe the synchronous nature of $\mathrm{CR}$ and the management of the show by a community member increases the agency of refugees in contrast to asynchronous systems. This is due to CR negating the need for third party mediation and content editing. The creation of video documentaries within the Za'atari Syrian refugee camp surfaced the conflict between NGO agendas and community realities [1]. Therefore, the agendas of third party mediators may eventually dictate the content of asynchronous refugee community radio shows.

\subsection{Community Radio Shows \& Privacy}

The congregation of the women around one listener phone shed light on privacy issues within the context of health community radio shows. In previous work [23], the women were comfortable discussing their health concerns in focus groups, however, during the running of the shows tensions arose regarding discussing health issues in front of specific community members. Familial influences and disputes, such as that of the great mother-in-law, were prominent in regards to privacy preferences and are typical of such contexts [14]. Like other studies investigating privacy and health information [12], fear of abuse of information by other members led participants to reconsider the level of preferred anonymity and privacy. Tensions that arose regarding privacy also implied the preference for selecting the group of individuals with whom a listener would want to share a radio show. Although this may further deepen existing divides within the community, the creation of multiple community radio shows based on preference 
improves comfort and interest in the shows which is deemed a success [6].

\subsection{Community Radio Shows for Trust \& Understanding}

Our findings support previous literature, that emphasize the importance of listeners trusting the guest within such health education mediums [13]. This is vital given that war has been found to erode societal trust between communities and institutions [21]. Our data show that the refugee participants found the information disseminated by the HCPs to be reliable, so much so that they perceived them to be better than the HCPs they currently visit. This is supported by their request for the HCPs' names so they might visit their clinics. Similar to other forms of digital communication [31], HCPs found the high level of interaction among the refugee community as key in their evaluation of the engagement and in building trust [31]. HCPs reported that they trusted that the women were actively listening to the shows and the host was a reliable mediator. Indeed, they placed their trust in the host in organizing the show, and allowed her to successfully manage the Q\&A segments without attempting to dominate the facilitation of the shows. Building and leveraging this mutual trust between HCPs and communities may contribute to the sustainability of such community engagements [15].

Lastly, while community radio shows allow for the generation of culturally and community relevant content $[6,16,18,19]$, our findings showed that community radio shows can bring about a change in HCP perspectives regarding refugee communities, through increasing understanding. The high engagement of refugees with HCPs instigated a shift from viewing refugees as a low health literate community, to that of a community that is health aware. Additionally, these engagements provided a window into the context of refugees thus increasing HCPs' understanding of the constraints refugees experience regarding their health. This understanding could improve health delivery and communication to refugee populations.

\section{CONCLUSION}

The effect of peer-led community radio shows on community dynamics has not previously been explored, especially when applying IVR technologies in resource constrained communities. The potential for synchronous community radio shows to improve health outreach and education was highlighted. Furthermore, we displayed the effect of community radio shows on community agency and on HCP-refugee relationships. We were also able to investigate aspects that need to be considered when designing for future work with an emphasis on considering shifts in community dynamics when deploying such technologies.

\section{ACKNOWLEDGEMENTS}

This work was funded by an EPSRC award EP/L016176/1 (Centre for Doctoral Training in Digital Civics). Data supporting this publication is not openly available due to confidential considerations. Access may be possible under appropriate agreement. Additional metadata record at http://dx.doi.org/10.17634/154300-47. Please contact Newcastle Research Data Service at rdm@ncl.ac.uk for further information or access requests. We would like to acknowledge the refugee community that welcomed us, the aid of Beyond Association, whose representative accompanied us to the ITSs, and the Lebanese Ministry of Public Health, for allowing us to access the population.

\section{REFERENCES}

[1] Jens Adam Hedegaard Hansen, Charlotte Erhardtsen, Sofia Lin Mogensen, Mia Holbek and Guorun Henrysdottir. 2014. Power relations in the Za ' atari refugee camp. Bachelor's Dissertation. Roskilde University, Roskilde, Denmark. OAI identfier: oai:rudar.ruc.dk:1800/16455

[2] Jennifer Baranoff, R. Israel Gonzales, Jay Liu, Heidi Yan, and Jimin Zheng. 2015. Lantern: Empowering Refugees Through Community-Generated Guidance Using Near Field Communication. In Proceedings of CHI'15 Extended Abstracts (CHI'15). ACM, New York, NY, 7-12.

[3] Virginia Braun and Victoria Clarke. 2006. Using thematic analysis in psychology. Qualitative Research in Psychology 3, 2, 77-101. DOI: http://doi.org/10.1191/1478088706qp063oa

[4] Deana Brown and Rebecca E Grinter. 2016. Designing for Transient Use: A Human-in-the-loop Translation Platform for Refugees. In Proceedings of the 2016 CHI Conference on Human Factors in Computing Systems (CHI'16). ACM, New York, NY, 321-330.

[5] Robert Chambers. 2006. Participatory mapping and geographic information systems: whose map? Who is empowered and who disempowered? Who gains and who loses? The Electronic fournal of Information Systems in Developing Countries 25, 2, 1-11. DOI: http://doi.org/144.214.55.140

[6] Chris Csíkszentmihályi and Jude Mukundane. 2016. RootIO: ICT + Telephony for Grassroots Radio. IST-Africa Week Conference, IEEE, 1-13.

[7] Nicola Dell and Neha Kumar. 2016. The Ins and Outs of HCI for Development. In Proceedings of the 2016 CHI Conference on Human Factors in Computing Systems (CHI'16). ACM, New York, NY, 312-318.

[8] Joseph Finkelstein and Jeffrey Wood. 2011. Information technology for continuous patient health education. In Proceedings of the 2011 conference on Information technology education (SIGITE '11). ACM, New York, NY, 245-250.

[9] Karen E Fisher, Katya Yefimova, and Eiad Yafi. 2016. "Future's Butterflies:" Co-Designing ICT Wayfaring Technology with Refugee Syrian Youth. In Proceedings of ACM SIGCHI Conference on Interaction Design and Children 2016 (IDC'16). ACM, New York, NY, 25-36.

[10] Marie Gillespie, Lawrence Ampofo, Margaret Cheesman, Becky Faith, Evgenia Iliadou, Ali Issa, Souad Osseiran, Dimitris Skleparis. 2016. Mapping Refugee Media fourneys Smartphones and Social Media Networks Research Report. $\begin{array}{llll}\text { Retrieved August } & 23, & 2016 & \text { from }\end{array}$ http://www.open.ac.uk/ccig/sites/www.open.ac.uk.ccig/files/Mapping Refugee Media Journeys 16 May FIN MG_0.pdf

[11] Felicity Goodyear-Smith and Stephen Buetow. 2001. Power issues in the doctor-patient relationship. Health Care Analysis 9, 4, 449-462. DOI: http://doi.org/10.1023/A:1013812802937

[12] T. Ryan Hoens, Blanton Marina, and Nitesh V. Chawla. 2013. Reliable medical recommendation systems with patient privacy. ACM Transactions on Intelligent Systems and Technology. 4, 4, 1-31. DOI: http://doi.org/10.1145/2508037.2508048

[13] Konstantinos Kazakos, Siddhartha Asthana, Madeline Balaam, Mona Duggal, Amey Holden, Limalema Jamir, Nanda Kishore Kannuri, Saurabh Kumar, Amarendar Reddy Mamindla, Subhashini Arcot Manikam, GVS Murthy, Papreen NAhar, Peter Phillimore, Shreyaswi Sathyanath, Pushpendra Singh, Meenu Singh, Pete Wright, Deepika Yadav and Patrick Olivier.2016. A RealTime IVR Platform for Community Radio. In Proceedings of the $2016 \mathrm{CHI}$ Conference on Human Factors in Computing Systems (CHI'16). ACM, New York, NY, 343-354. 
[14] Neha Kumar and Richard J. Anderson. 2015. Mobile Phones for Maternal Health in Rural India. In Proceedings of the 33rd Annual ACM Conference on Human Factors in Computing Systems (CHI '15). ACM, NEw York, NY, 427-436.

[15] Neha Kumar, Waylon Brunette, Nicola Dell, Trevor Perrier, Beth Kolko, Gaetano Borriello and Richard Andrerson . 2015. Understanding sociotechnical implications of mobile health deployments in India, Kenya, and Zimbabwe. Information Technologies \& International Development 11, 4, 17-32.

[16] Aparna Moitra, Vishnupriya Das, Gram Vaani, Archna Kumar, and Aaditeshwar Seth. 2016. Design Lessons from Creating a Mobile-based Community Media Platform in Rural India. In Proceedings of the Eighth International Conference on Information and Communication Technologies and Development (ICTD '16). ACM, New York, NY, 14-25.

[17] Maletsabisa Molapo and Gary Marsden. 2013. Software support for creating digital health training materials in the field. In Proceedings of the Sixth International Conference on Information and Communication Technologies and Development (ICTD '13). ACM, New York, NY, 205-214.

[18] Preeti Mudliar, Jonathan Donner, and William Thies. 2012. Emergent Practices Around CGNet Swara , A Voice Forum for Citizen Journalism in Rural India Categories and Subject Descriptors. In Proceedings of the Fifth International Conference on Information and Communication Technologies and Development (ICTD'12). ACM, New York, NY, 159-168.

[19] Neil Patel, Deepti Chittamuru, Anupam Jain, Paresh Dave, and Tapan S. Parikh. 2010. Avaaj Otalo - A Field Study of an Interactive Voice Forum for Small Farmers in Rural India. In Proceedings of the 28th international conference on Human factors in computing systems (CHI '10). ACM, Newy York, NY, 733-742.

[20] Agha Ali Raza, Farhan Ul Haq, Zain Tariq, et al. 2013. Job opportunities through Entertainment: Virally Spread Speech-Based Services for Low-Literate Users. In Proceedings of the SIGCHI Conference on Human Factors in Computing Systems (CHI '13). ACM, New York, NY, 2803-2812.

[21] Bryan Semaan and Gloria Mark. 2011. Technology-Mediated Social Arrangements to Resolve Breakdowns in Infrastructure During Ongoing Disruption. ACM Transactions on Computer-Human Interaction (TOCHI) 18, 4, 1-21. DOI: http://dx.doi.org/10.1145/2063231.2063235

[22] Jahanzeb Sherwani, Nosheen Ali, Sarwat Mirza, Anjum Fatma, Yousuf Memon, Mehtab Karim, Rahul Tongia and Roni Rosenfeld. 2007. Healthline: Speechbased access to health information by low-literate users. In Proceedings of International Conference on Information and Communication Technologies and Development (ICTD'07). IEEE, 1-9.

[23] Reem Talhouk, Sandra Mesmar, Anja Thieme, Madeline Balaam, Patrick Olivier, Chaza Akik, Hala Ghattas. 2016. Syrian Refugees and Digital Health in Lebanon: Opportunities for Improving Antenatal Health. In Proceedings of the 2016 CHI Conference on Human Factors in Computing Systems (CHI'16). ACM, Newy York, NY, 331-342.

[24] Priyamvada Tiwari and Keyur Sorathia. 2014. Visualising and systematizing a per-poor ICT intervention for Rural and Semi-urban Mothers in India. In Proceedings of the 7th International Symposium on Visual Information Communication and Interaction (VINCI '14). ACM, New York, NY, 129-138.

[25] United Nations High Commissioner for Refugees. 2016. Syria Regional Refugee Response - Regional Overview. Retrieved August 23, 2016 from http://data.unhcr.org/syrianrefugees/regional.php

[26] Aditya Vashistha, Edward Cutrell, Gaetano Borriello, and William Thies. 2015. Sangeet Swara: A Community-Moderated Voice Forum in Rural India. In Proceedings of the 33rd Annual ACM Conference on Human Factors in Computing Systems (CHI '15). ACM, New York, NY, 417-426.

[27] Aditya Vashistha and William Thies. 2012. IVR junction: Building scalable and distributed voice forums in the developing world. Retrieved August 23, 2016 from https://www.usenix.org/system/files/conference/nsdr12/nsdr12-final4.pdf

[28] Anna Weibert, Konstantin Aal, Thomas von Rekowski, and Volker Wulf. 2015 Hey, can we make that, please?: Upon Craft as a Means for Cross-cultural Community-Building. Community Informatics 11, 2.

[29] Ying Xu, Carleen Maitland, and Brian Tomaszewski. 2015. Promoting Participatory Community Building in Refugee Camps with Mapping Technology. In Proceedings of the Eighth International Conference on Information and Communication Technologies and Development (ICTD '15). ACM, New York, NY, Article 67, 4 pages.

[30] George Yerousis, Konstantin Aal, Thomas von Rekowski, David W. Randall, Markus Rohde, and Volker Wulf. 2015. Computer-Enabled Project Spaces. In Proceedings of the 33rd Annual ACM Conference on Human Factors in Computing Systems (CHI '15). ACM, New York, NY, 3749-3758.

[31] Jun Zheng, Elizabeth Veinott, Nathan Bos, Judith S Olson, and Gary M Olson. 2002. Trust without Touch: Jumpstarting long-distance trust with initial social activities. In Proceedings of the SIGCHI Conference on Human Factors in
Computing Systems (CHI ’02). ACM, New York, NY, 141-146. 